\title{
Development of Converged Device-Based Exercise Program for Preventing Fall and Increasing Physical Activity for the Elderly
}

\author{
Sunyoung Kang \\ Department of Physical Education, Korea University, Seoul, Korea \\ E-mail:1010kang@hanmail.net
}

Received 28 September 2020; Accepted 31 October 2020;

Publication 15 February 2021

\begin{abstract}
As the effects of COVID-19, many changes are occurring in the daily life. Breaking away from the temporal and spatial restrictions, the exercise method utilizing converged device in non-contact fashion is emerging. In the present study, home training with utilization of the converged device for fall prevention and improvement of daily life in behalf of the frail elderly has been composed, and execution process designed. Converged device-based exercise program extracted through Delphi analysis is composed of essential 8 types of motion reflecting the performance capability of the frail elderly as the subject, though easy, have been selected. Converged device-based exercise program configured the system in a structure of subject, interface, and administrator for the purpose of utilizing this exercise program. Overall execution process is composed 3 stages, and implemented with the elderly and the trainer being converged via medium. For the overall implementation, the elderly performs the exercise program under leading of the trainer as the administrator. Depending on the condition of the elderly as the subject, the trainer selects the difficulty of exercise, which the elderly performs and implements the exercise program while communicating with the trainer. The converged device-based exercise program that is applicable to the elderly as
\end{abstract}

Journal of Web Engineering, Vol. 20_1, 103-112.

doi: 10.13052/jwe1540-9589.2015

(C) 2021 River Publishers 
a digitally vulnerable class is expected to bring about not only fall prevention and increased physical activities but also subsidiary effects of producing digital device-friendly environments for the elderly as a digitally vulnerable class.

Keywords: Converged device-based, the frail elderly, fall, interface.

\section{Introduction}

Because of the effects of COVID-19, enjoying exercises by following the instructor's motion in the screen at a comfortable place and convenient time while looking into the Youtube or mobile phone app is becoming a routine scent that can be frequently encountered in the surroundings. As the home training that can be enjoyed by anyone in his/her own house breaking away from the temporal and spatial constraints is becoming popular, the interests in the related technologies are also being increased consistently.

Due to the changes in population structure and a rapid increase in average expected lifetime, the importance of increased independence and active selfhealth care of the elderly for improvement of life quality in old age is being increased. While old people are feeling the threat to health because of the physical changes caused by aging, among which the fall of an elderly that occurs together with changes in musculoskeletal system is being considered as the most dangerous accident among the factors threatening the quality of life particularly in later years [1]. Regular exercise has been shown to reduce the risk of falls by $20-35 \%$, and brings about the increase of daily life and exercise capability [2,3]. In a study for the subject of elderly people of more than 80 years in age, exercises for improvement of muscular strength and balancing capability is reported to have reduced the risks of fall [4]. Therefore, regular and appropriate exercises are required to reduce the fall risks and improve the performance capability of daily life.

Since old people are very vulnerable in mobility and activity aspects, they can be considered as a class that is very alienated from contents experience of diverse physical activities. Although the importance of physical activities is continuously emphasized for them, there is much realistic difficulty in active participation in physical activities that are suited to them and enjoyable as well [5]. Physical activity contents are not diversified that can satisfy internal motivation such as fun and pleasure as the most important factor in continued participation in physical activities. 
Converged Device-Based Exercise Program where the technology for reproduction in the screen of diverse types of multimedia such as text, video, audio, painting, picture, animation, etc. are grated to the technology allowing motion recognition to recognize the elderly's physical activities and store data induces fun with synthesis of interest and curiosity so that the effects of providing self-motivation can be expected. Through development of Converged Device-Based Exercise Program that is applicable to elderly people as the digitally vulnerable class, not only fall prevention and increased physical activities but also subsidiary effects of producing digital device-friendly environments are expected to be produced.

In the present study, changes in paradigm for elderly's physical activities will be proposed by composing the Converged Device-Based Exercise Program and presenting its utilization measures for fall prevention and increased physical activities in behalf of the frail elderly.

\section{Contents of Fall Prevention and Physical Activity Increase Program in the Frail Elderly}

The frail elderly is the aged with vulnerable physical functions and cognitive functions so as to have lowered daily life activities such as going out, etc. Because of degradation in physical activities, muscular strength and muscle mass are reduced, dropping stamina and increasing proneness to occurrence of chronic diseases. To minimize the health problems of these frail elderly and improve their health, active intervention is needed [6]. Regular exercises and physical activities are being recommended along with the improvement methods for muscular strength or flexibility, balance by using appropriate exercise methods $[7,8]$.

Through collection, analysis and case study of the data related to fall prevention and physical activities of the frail elderly, cases applicable to the field were analyzed for securing of diverse empirical evidences and program contents, and Delphi analysis was executed for 3 times based on the secures exercise program contents. For the specialists selected by purposive sampling of elderly, official related to physical education policies, professor of physical education study, specialist related to elderly's physical education, engineer related to converged exercise device technology, etc. diverse opinions concerning fall prevention and increased physical activities were derived. Through this, the contents of converged device-based exercise program were extracted as self-initiated and creative physical activities (Figure 1). 


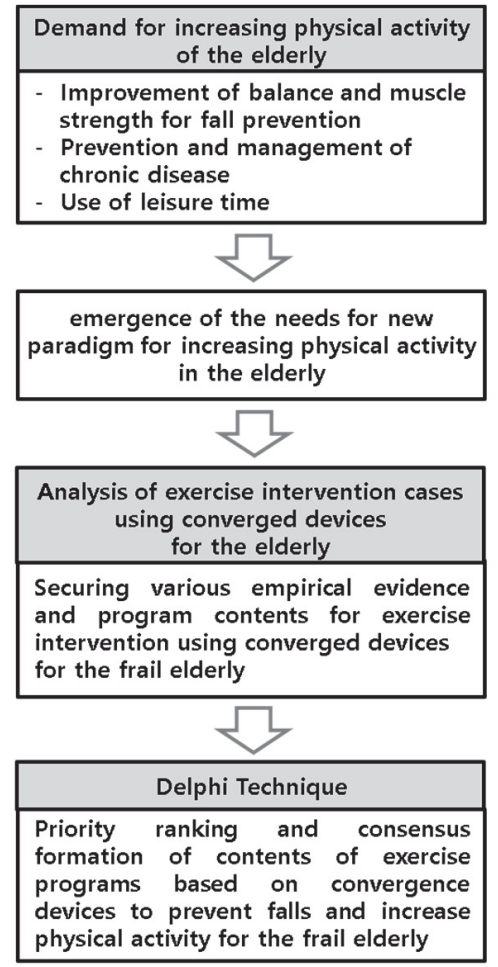

Figure 1 Composition process of exercise content that help prevent falls and increases physical activity.

Converged device-based exercise program extracted through Delphi analysis is composed of the leg muscle strength exercise and the balance exercise which are effective for fall prevention. Essential 8 types of motion reflecting the performance capability of the frail elderly as the subject, though easy, have been selected. These motions include motion helpful for taking posture, motion strengthening balance, motion training muscle groups for posture maintenance, and motion training leg reinforcement, which were categorized into 4 areas with the contents shown in Figure 2.

\section{Configuration and Expansion of Converged Device-Based Exercise Program System}

Converged device-based exercise program configured the system in a structure of subject, interface, and administrator. This was planned for the purpose 


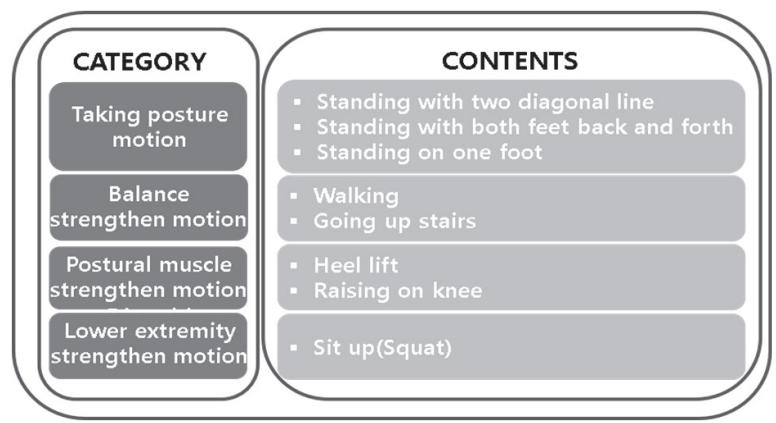

Figure 2 Converged device-based exercise program extracted through Delphi analysis.

of utilizing the exercise program to prevent fall and increase physical activities for the frail elderly as derived through collection of specialist opinions.

Elderly is the principal agent who performs the exercise program through interface as the subject as well as the executer. The interface is a medium between the elderly and the trainer, allowing implementation of the exercise program by utilization of PC or mobile device. The administrator is the trainer as an evaluator as well as helper of the elderly's exercise performing capability, helping to enable safe performance of the exercise program suited to elderly's current health state. The trainer proposes the exercise program of optimum intensity by evaluation of family history, illness history, stamina, and exercise functions of the elderly, and provides feedbacks after the elderly's execution of program. Interface is involved in the execution of exercise program, and takes charge of the role of providing real-time feedbacks to both the elderly and the trainer (Figure 3).

Overall execution process is composed 3 stages as shown in Figure 4.

- Step 1: Trainer as the administrator personally visits the house by considering the characteristics of the frail elderly with degradation of daily life activities, and collects basic data through consultation with the subject. Tests are performed for individual characteristics such as the subject's life style, family history, illness status, etc. and basic stamina of the frail elderly, motion capability in daily life, static balance, exercise functions such as combined movement capability, etc. Through this, comprehensive exercise performing capabilities of the subject are determined.

- Step 2: While receiving the trainer's assistance after setting of the intensity suited to the subject's exercise capability that has been evaluated in 


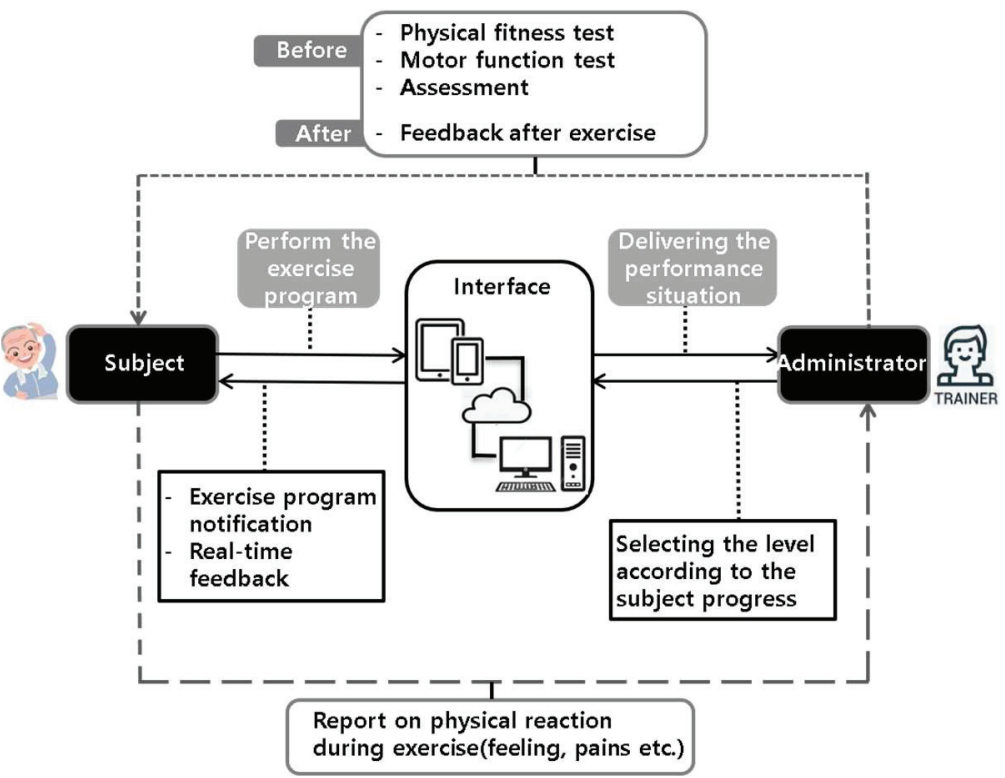

Figure 3 Composition diagram of exercise program system.

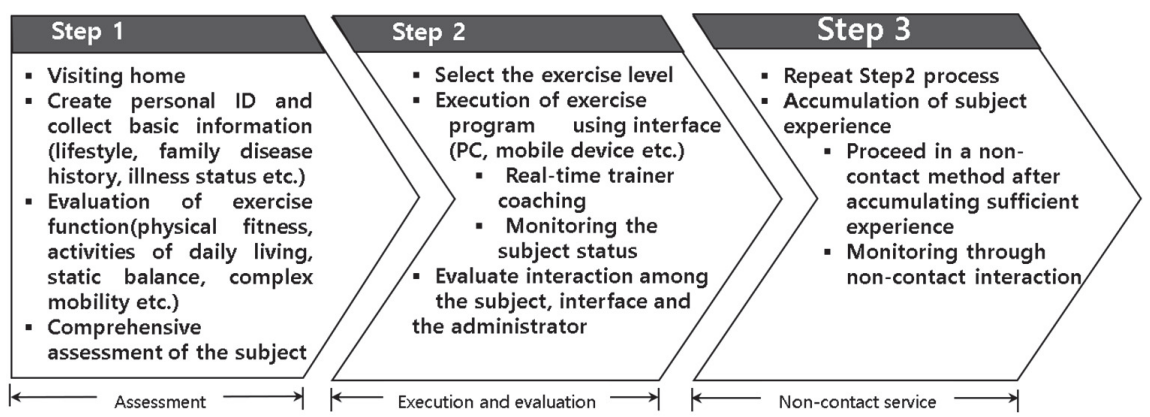

Figure 4 Execution process of exercise program system.

Step 1, simulation is implemented. Movements are collected through motion sensor and pressure sensor, which is reflected in the real-time performance situation of the exercise program. During the simulation, the trainer performs real-time observation of overall situations, monitoring the subject's stamina situation and movements. When exercise data is collected through this, self-monitoring is possible and the trainer is provided the data capable of managing the subject's exercise capability. When the simulation is concluded, it is evaluated whether or 


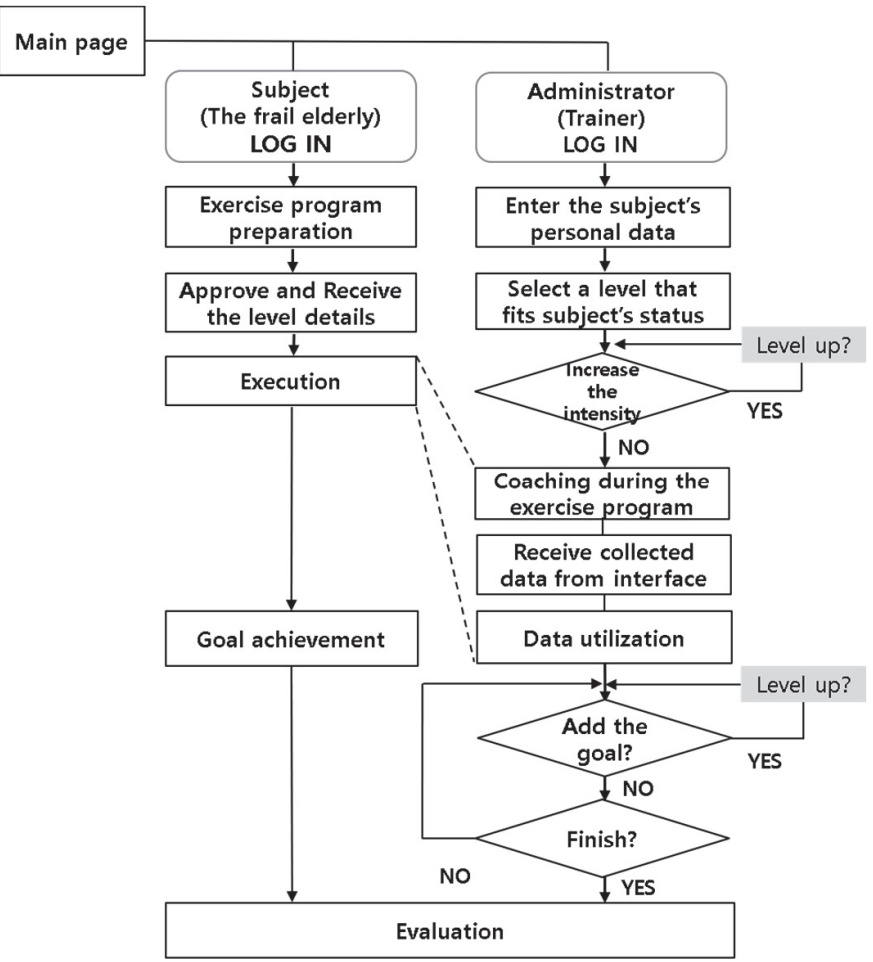

Figure 5 Progress process of the converged device-based exercise program system.

not interactions among the subject, interface and the administrator are realized smoothly, and the opinion about the subject's use experience is listened to.

- Step 3: To accumulate the subject's use experience, the trainer pays visits at a given interval to repeatedly implement the process of Step 2. When meaningful user experience is accumulated to the extent that the subject himself/herself can execute the program, no more visits by the trainer are realized and non-contact method is implemented.

Detailed execution scenario of the program is implemented with the elderly and the trainer being converged via medium as shown in Figure 5. For the overall implementation, the elderly performs the exercise program under leading of the trainer as the administrator. Depending on the condition of the elderly as the subject, the trainer selects the difficulty of exercise, which the elderly performs and implements the exercise program while communicating with the trainer. 


\section{Conclusion}

As the effects of COVID-19, many changes are occurring in the daily life. Breaking away from the temporal and spatial restrictions, the exercise method utilizing converged device in non-contact fashion is emerging. In the present study, home training with utilization of the converged device for fall prevention and improvement of daily life in behalf of the frail elderly has been composed, and execution process designed. By inducing the elderly to allow participation in physical activities by diverse and fun methods, accumulating the appearance with elderly's physical activity capabilities as data, and having the data checked continuously, the elderly's fall prevention and increased physical activities and further contents provision and big data utilization for health improvement will become possible. Through exercise intervention method of the new paradigm, through experience and operation of the social technology, new technology along with pleasure that could not be easily obtained from the exercise of usual participation, the elderly's curiosity and fun will be stimulated more than by any other educational means. This will lead to increased physical activities and comprehensive changes of behavior, enabling provision of the opportunities for improvement of stamina and health. In addition, the converged device-based exercise program that is applicable to the elderly as a digitally vulnerable class is expected to bring about not only fall prevention and increased physical activities but also subsidiary effects of producing digital device-friendly environments for the elderly as a digitally vulnerable class.

\section{Acknowledgements}

This work was supported by the Ministry of Education of the Republic of Korea and the National Research Foundation of Korea (NRF2019S1A5B5A07107217).

\section{References}

[1] S. D. Berry, R. R. Miller, 'Falls: Epidemiology, pathophysiology, and relationship to fracture', Current Osteoporosis Reports, 6(4), pp. 149154, 2008.

[2] M. J. Daley, W. L. Spinks, 'Exercise, mobility and aging, Sports medicine', 29(1), pp. 1-12, 2000. 
[3] K. M. Means, D. E. Rodell, P. S. O'Sullivan, 'Balance, mobility, and falls among community-dwelling elderly persons: effects of a rehabilitation exercise program', American journal of physical medicine \& rehabilitation, 84(4), pp. 238-250, 2005.

[4] M. M. Gardner, D. M. Buchner, M. C. Robertson, A. J. Campbell, 'Practical implementation of an exercise-based falls prevention program', Age and ageing, 30(1), pp. 77-83, 2001.

[5] I. S. Park, 'Role of Exercise on Fall Prevention for Health Management in the Elderly', Review of Korea Entertainment Industrial Association, 10(6), pp. 343-350, 2016.

[6] Y. I. Park, K. Y. Lee, T. I. Kim, M. H. Jeon, D. O. Kim, J. H. Kim, 'The effects of exercise in the frail elderly', J Korean Acad Community Health Nurs., 23(1), pp. 91-101, 2012.

[7] K. Rockwood, X. Song, C. MacKnight, H. Bergman, D. B. Hogan, I. McDowell, A. Mitnitski, 'A global clinical measure of fitness and frailty in elderly people', Canadian Medical Association Journal, 173, pp. 489495, 2005.

[8] C. J. Jones, D. J. Rose, 'Physical activity instruction of older adults'. Champaign. IL: Human Kinetics, 2005.

\section{Biography}

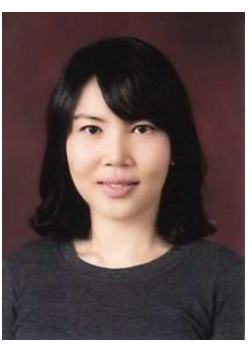

Sunyoung Kang received her Ph.D. degree in the department of physical education from Korea University, Seoul, Korea, in 2005. Her research interests include sports science, exercise prescription, wellness IT, and rehabilitation engineering. 
